\title{
ATRIBUIÇÕES DO ENFERMEIRO AO PACIENTE COM COMPLICAÇÕES RENAIS PÓS COVID-19 EM UTI: REVISÃO DE ESCOPO
}

\author{
NURSES ATTRIBUTIONS TO PATIENTS WITH KIDNEY COMPLICATIONS AFTER COVID-19 IN ICU:
}

\author{
SCOPING REVIEW
}

ATRIBUCIONES DE LAS ENFERMERAS A PACIENTES CON COMPLICACIONES RENALES DESPUÉS DEL COVID-19 EN UCI: REVISIÓN DEL ALCANCE

\author{
Maressa Samai Pinheiro Silva Smith ${ }^{1 * i D}$ \\ Letycia Luciano Lucena Alves ${ }^{1}$ (D) \\ Camila Priscila Abdias do Nascimento ${ }^{2}$ (D)
}

\begin{abstract}
${ }^{1}$ Enfermeira graduada pela Universidade Potiguar (UnP), Natal, Rio Grande do Norte, Brasil; ${ }^{2}$ Mestre e doutoranda em Enfermagem Programa de Pós-Graduação em Enfermagem-PGENFda Universidade Federal do Rio Grande do Norte-UFRN, Natal, Rio Grande do Norte, Brasil.*Autor correspondente: maressa-samai@hotmail.com.
\end{abstract}

Recebido: 12/11/2021 | Aprovado: 09/12/2021 | Publicado: 15/12/2021

\begin{abstract}
Resumo: Evidenciar as contribuições da assistência prestada pelo enfermeiro ao indivíduo com complicações renais correlacionadas a infecção causada pelo COVID-19. O estudo trata-se de uma revisão de escopo (Scoping Review), caracterizada como um tipo de delineamento que apresenta como objetivo esclarecer e mapear os principais conceitos que alicerçam a pesquisa. A revisão seguiu as instruções inclusas no manual de revisões do Joanna Briggs Institute (JBI), de 2020, sendo realizada através de consulta online nas seguintes bases de dados: Scientific Eletronic Library Online (SciELO), Literatura Latino Americana e do Caribe em Ciências da Saúde (LILACS) e Biblioteca Virtual em Saúde do Ministério da Saúde (BVSMS). Inicialmente foram encontrados 437 artigos, destes, 16 foram selecionados para compor a amostra final. Dentre os agravos decorrentes da infecção pelo novo coronavírus, é notório que a ocorrência de lesão renal aguda (LRA), possui intrínseca relação com o COVID-19 e está associada a um quadro de pior progressão clínica e/ou mortalidade do indivíduo. Assim, a ampla frequência de pacientes com LRA nas unidades de terapia intensiva exigem do enfermeiro, um papel fundamental, baseado em uma intervenção que proporcionem qualidade e segurança. E este, juntamente aos demais profissionais da saúde que compõem uma equipe multidisciplinar comprometidos com a assistência nefrológica devem conceder os cuidados necessários aos pacientes pelos quais são responsáveis alicerçados nos princípios assistenciais. Tornando-se evidente a relevância do papel desempenhado pelo profissional enfermeiro centrado no cuidado físico abrangendo similarmente fatores biopsicossociais, na busca pela promoção da saúde e bem-estar do paciente no contexto inserido.
\end{abstract}

Palavras-chave: Enfermagem. COVID-19. Injúria Renal Aguda. Unidade de Terapia Intensiva.

Abstract: Show the contributions of care provided by nurses to individuals with renal complications correlated to infection caused by COVID-19. The study is a scope review (Scoping Review), characterized as a type of design that aims to clarify and map the main concepts that underlie the research. The review followed the instructions included in the Joanna Briggs Institute (JBI) Review Manual, 2020, and was carried out through online consultation in the following databases: Scientific Electronic Library Online (SciELO), Latin American and Caribbean Literature in Sciences of the Health (LILACS) and the Ministry of Health's Virtual Health Library (BVSMS). Initially, 437 articles were found, of which 16 were selected to compose the final sample. Among the problems resulting from infection by the new coronavirus, it is notorious that the occurrence of acute kidney injury (AKI) has an intrinsic relationship with COVID-19 and is associated with worse clinical progression and/or individual mortality. Thus, the wide frequency of patients with AKI in intensive care units requires the nurse to play a fundamental role, based on an intervention that provides quality and safety. And this, together with other health professionals who make up a multidisciplinary team committed to nephrological care, must provide the necessary care to patients for whom they are responsible, based on care principles. The relevance of the role played by the professional nurse, centered on physical care, is evident, covering similarly biopsychosocial factors, in the search for the promotion of the patient's health and well-being in the inserted context. 
Keywords: Nursing. COVID 19. Acute Kidney Injury. Intensive Care Units.

Resumen: Muestre las contribuciones de la atención brindada por enfermeras a personas con complicaciones renales correlacionadas con la infección causada por COVID-19. El estudio es una revisión de alcance (Scoping Review), caracterizado como un tipo de diseño que tiene como objetivo aclarar y mapear los principales conceptos que subyacen a la investigación. La revisión siguió las instrucciones incluidas en el Manual de Revisión del Intituto Joanna Briggs (JBI), 2020, y se realizó mediante consulta en línea en las seguientes bases de datos: Scientific Electronic Library Online (SciELO), Literatura Latinoamericana y Caribeña en Ciencias de la Salud (LILACS) y la Biblioteca Virtual en Salud del Ministerio de Salud (BVSMS). Inicialmente se encontraron 437 artículos, de los cuales 16 fueron seleccionados para conformar la muestra final. Entre los problemas derivados de la infección per el nuevo coronavirus, es notorio que la ocurrencia de daño renal agudo (IRA) tiene una relación intrínseca con el COVID -19 y se asocia a una peor progresión clínica y/ o mortalidad en el individuo. Así la gran frecuencia de pacientes con LRA en unidades de cuidados intensivos obliga al enfermero a jugar un papel fundamental, basado en una intervención que aporte calidad y seguridad. Y esto, junto con otros profesionales de la salud que integran un equipo multidisciplinar comprometido con la atención nefrológica, deben brindar la atención necesaria a los pacientes de los que son responsables, basados en principios asistenciales. Es evidente la relevancia del papel desempeñado por la enfermera profesional, centrada en el cuidado físico, abarcando igualmente factores biopscosociales, en la búsqueda de la promoción de la salud y el bienestar del paciente en el contexto insetado.

Palabras-clave: Atención de Enfermería. COVID-19. Lesión Renal Aguda. Unidades de Cuidados Intensivos.

\section{INTRODUÇÃO}

Declarada oficialmente como pandemia em março de 2020 pela Organização Mundial de Saúde (OMS), a doença ocasionada pelo novo coronavírus foi denominada posteriormente como Severe Acute Respiratory syndrome Coronavírus-2 (SARS-CoV-2), causando a doença chamada de Corona Vírus Disease-19 (COVID-19). Esta, apresenta alta taxa de transmissibilidade entre humanos através de gotículas respiratórias liberadas no ambiente por um indivíduo infectado. Com a heterogeneidade e gravidade dos sintomas, diversos pacientes infectados pelo novo coronavírus necessitaram de internação em unidades de terapia intensiva (UTI) (Nunes, 2020).

A evolução clínica da infeção originada pela doença pode fomentar uma série de condições patológicas sendo capaz de manifestar menor ou maior gravidade a depender de cada indivíduo. Possuindo potencial para desencadear sintomas respiratórios leves ou até mesmo pneumonia, lesões cardíacas, Síndrome da Angústia Respiratória Aguda (SARA), sepse e choque séptico. Podendo igualmente suscitar o desenvolvimento de complicações renais influenciadas pelas condições e fatores de risco do indivíduo tendo potencial risco de acarretar o óbito por meio desta enfermidade (Brasil, 2020).

Perante a influência que o novo coronavírus determina sobre os sistemas corporais provocando disfunções orgânicas, as complicações renais como Lesão Renal Aguda (LRA) expressam alta relevância no contexto da morbimortalidade, apontando preocupação quanto a clínica e prognóstico de pacientes com comorbidades pré-existentes como a Doença Renal Crônica (DRC) e doença renal em estágio terminal (DRET). No Brasil, é crescente o número da morbidade e mortalidade por lesão renal, apenas no ano de 2019 foram registradas 121.581 internações, apresentando uma taxa de letalidade de aproximadamente 12,40\%, totalizando 15.080 óbitos (Datasus, 2019; Ulu et al., 2020; Pecly et al., 2021).

No tocante as complicações renais em pacientes com COVID-19, um estudo de caso retrospectivo inicial desenvolvido na província de Hubei, China, descreveu uma incidência de LRA de cerca de 13,1\%, 
expondo preocupações iniciais quanto a relação da infecção pelo coronavírus e o sistema renal. Por sua vez, a associação entre COVID-19 e LRA no Brasil evidenciam dados sobre incidência de LRA em pacientes pós COVID-19 ainda iniciais, demonstrando a necessidade de observar o comportamento dessa fusão nos pacientes acometidos, a fim de otimizar as condutas terapêuticas e insumos necessários, com a finalidade de obter resultados clínicos positivos (Wang et al., 2020; Costa et al., 2020).

Por meio da análise deste cenário pandêmico, salienta-se que os pacientes em estado clínico crítico expõem maior susceptibilidade a desenvolver LRA, e por necessitarem de maiores cuidados intensivos a equipe de enfermagem detém importante contribuição na participação do cuidado direto a esse grupo por estarem também relacionados as complicações das Infecções Respiratórias Agudas (IRA). Diante dessa demanda, é relevante que a enfermagem inserida neste panorama apresente alta capacidade de atuação na prestação de cuidados dessa conjuntura. Assim sendo, é importante que o enfermeiro elabore estratégias para estabelecer treinamentos da equipe fundamentadas na segurança do paciente, uma vez que esse também é responsável pelo gerenciamento do cuidado, deve proporcionar uma assistência livre de danos (Queiroz \& Marques, 2020).

Nunes (2020) afirma que frente a essas condições clínicas, o enfermeiro contribui ativamente através da aplicação e manejo da terapia renal substitutiva (TRS), que compreende uma das alternativas de tratamentos realizados ao paciente com função renal prejudicada. Tornando imprescindível o alinhamento do fluxo de atendimento a esses pacientes, bem como a articulação entre demais profissionais que compõem uma equipe multidisciplinar com o propósito de atingir resultados almejados nos desfechos clínicos.

Mediante a relevância da atuação do profissional enfermeiro ao paciente crítico portador do coronavírus, o presente estudo possui como objetivo evidenciar as contribuições da assistência prestada pelo enfermeiro ao indivíduo com complicações renais correlacionadas a infecção causadas pelo COVID-19. Justificado pela necessidade de trazer maiores esclarecimentos sobre a temática, de modo a contribuir no conhecimento dos profissionais enfermeiros com o propósito de qualificar sua assistência.

\section{MATERIAL E MÉTODOS}

O estudo trata-se de uma revisão de escopo (Scoping Review), caracterizada como um tipo de delineamento que apresenta como objetivo esclarecer e mapear os principais conceitos que alicerçam a pesquisa. Bem como, identificar as lacunas existentes na literatura e analisar a natureza das produções compreendendo o modo que a pesquisa é conduzida na área em questão, além de selecionar estudos, extrair, sumarizar os dados, e realizar a difusão dos elementos da investigação (Cordeiro \& Soares, 2019; Peters et al., 2020).

A presente pesquisa foi elaborada de acordo com as instruções inclusas no manual de revisões do Joanna Briggs Institute (JBI), de 2020. Dando seguimento com a produção do protocolo de revisão de escopo, seguido do diagrama de fluxo em concordância com o Preferred Reporting Items for Systematic reviews and Meta Analyses extension 
for Scoping Reviews (PRISMA-ScR). Possuindo registro na plataforma Open Science Framework (OSF) (https://mfr.osf.io).

Nessa perspectiva, a questão norteadora foi baseada na estratégia mnemônica População, Conceito e Contexto (PCC) conforme recomendado pelo JBI. Sendo estabelecido: P - os profissionais enfermeiros; C - as atribuições ao paciente com complicações renais pós-COVID; C - em UTI. A partir dessa estratégia, formulouse a seguinte questão norteadora da pesquisa: Quais atribuições exercidas pelo enfermeiro ao paciente que encontra-se com complicações renais pós COVID-19 em uma UTI?

As pesquisas para a construção do estudo foram realizadas por meio de consultas online ao decorrer dos meses de setembro a outubro de 2021, por intermédio das seguintes bases de dados científicas: Scientific Eletronic Library Online (SciELO), Literatura Latino Americana e do Caribe em Ciências da Saúde (LILACS) e Biblioteca Virtual em Saúde do Ministério da Saúde (BVSMS). Os Descritores em Ciência da Saúde (DeCS) selecionados foram: "Enfermagem”. "COVID-19”; "Injúria Renal Aguda"; "Unidade de Terapia Intensiva”. Aplicando-se o operador boleano “AND” para combinação dos descritores.

A seleção dos estudos ocorreu de forma independente por dois pesquisadores da equipe, e para resolução de conflitos, um terceiro componentes deteve a autoridade na tomada de decisão na ausência de concordância. Como critérios de inclusão, os estudos analisados foram pré-selecionados após a leitura de seus respectivos títulos e resumos sendo mapeados de acordo com os autores, título, objetivo, tipo de estudo e nível de evidência científica, adotando a classificação da Agency for Healthcare Research and Quality (AHRQ) que engloba seis níveis: I- evidências resultantes de metanálise e revisão sistemática; II- evidências obtidas em ensaios clínicos com randomização; III- evidências obtidas em ensaios clínicos sem randomização; IV- evidências de estudos de coorte e de caso-controle; V- evidências oriundas de revisão sistemática de estudos descritivos e qualitativos; VIevidências baseadas em estudo descritivo ou qualitativo.

Foram estabelecidos como critérios de exclusão teses, dissertações e textos duplicados. Resultando em uma amostragem final de artigos disponíveis na íntegra e gratuitamente, publicados entre os meses de março de 2020 a março de 2021, elaborados nas línguas portuguesa, inglesa e espanhola.

\section{RESULTADOS}

Conforme os resultados levantados nas bases de dados científicas (BVSMS, LILACS e SciELO), foram identificados 437 artigos. Após a leitura dos estudos em sua integralidade e com a coleta de informações selecionadas quanto ao ano de publicação, título, resumo, tipo de estudo e objetivo da pesquisa, foram extraídos 16 estudos análogos aos critérios de elegibilidade dispostos na metodologia. Estes, seguiram sendo agrupados e apresentados em caráter descritivo respectivamente, através de fluxograma com a disposição quantitativa frente aos resultados da análise e por meio de tabela a fim de viabilizar a visualização dos resultados, conforme demonstrado na figura 1 e 2. 
Figura 1: Fluxograma do processo de seleção dos estudos adaptado do PRISMA- ScR. Natal, Rio Grande do Norte.

Brasil, 2021.
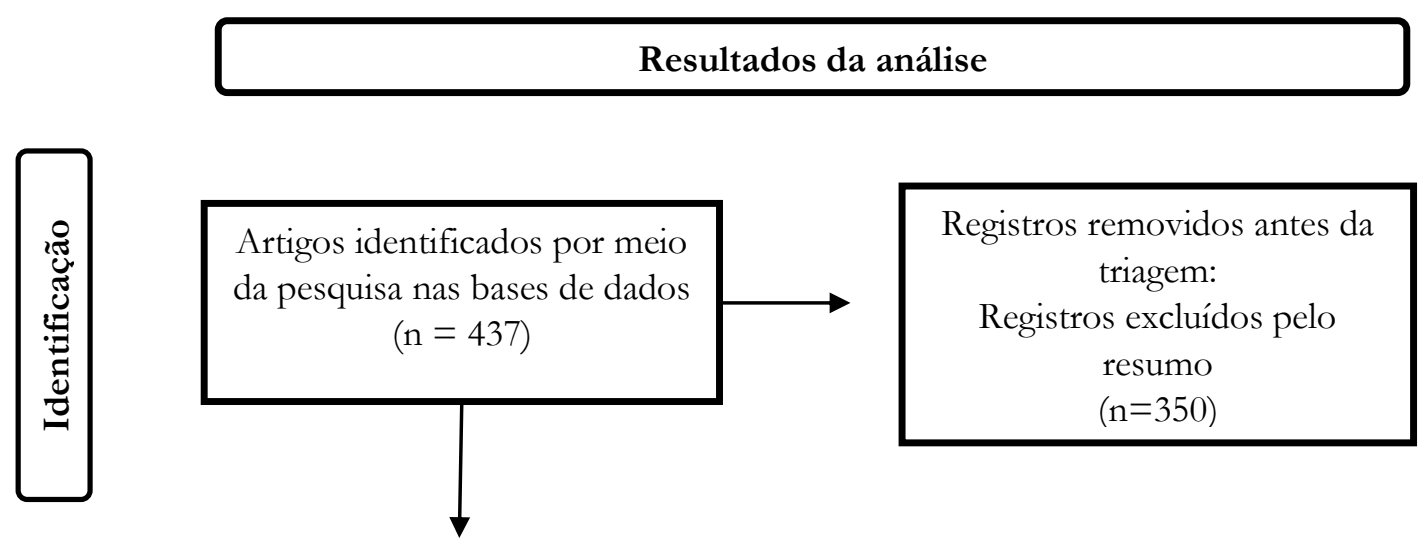
Registros removidos antes da triagem:
Registros excluídos pelo resumo $(\mathrm{n}=350)$
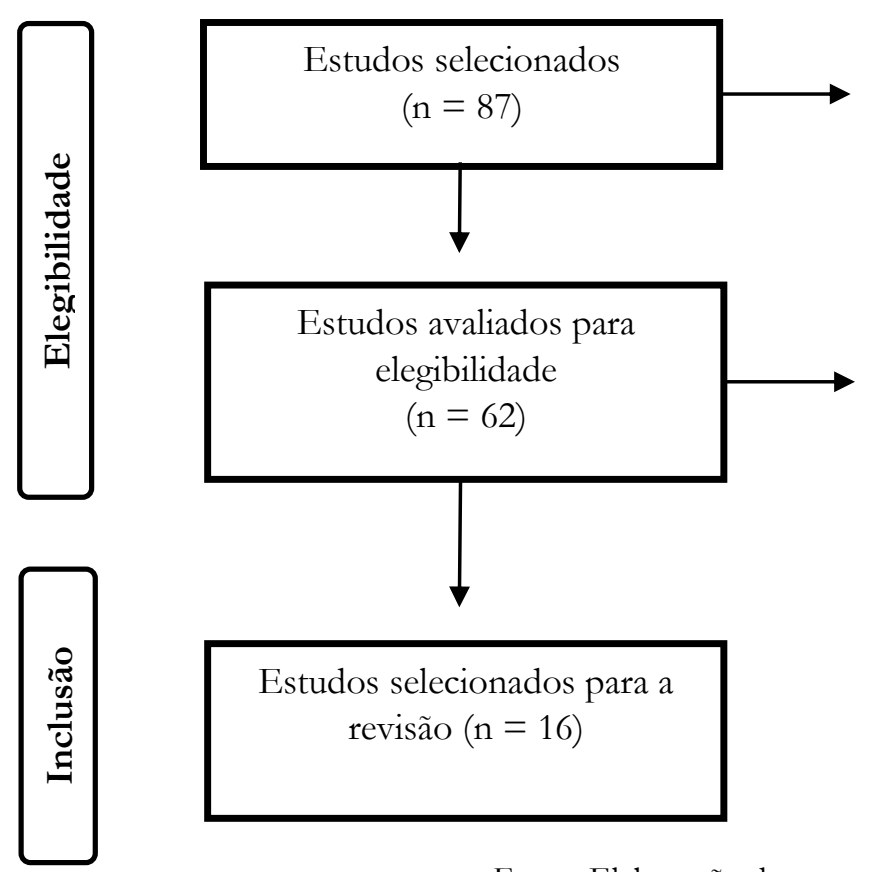

Estudos duplicados $(\mathrm{n}=25)$

Fonte: Elaboração dos autores, 2021.

Perante as análises oriundas dos estudos selecionados, evidencia-se que a maior parte foram desenvolvidos no ano de 2020 contendo 13 artigos, seguido dos anos de 2021 com 3 estudos. Quanto a origem da produção dos artigos, 12 foram elaborados na América do Sul, 03 na Ásia e 1 no continente europeu. Dentre os estudos apresentados, baseado nos níveis de evidência, observou-se que houve maior incidência em trabalhos com níveis de evidência V e VI com 31,25\% cada, seguidos dos níveis I apresentando 25\% e o IV com 12,5\%. 
Figura 2: Amostra dos resultados elencados para pesquisa. Natal, Rio Grande do Norte. Brasil, 2021.

\begin{tabular}{|c|c|c|c|c|}
\hline $\begin{array}{l}\text { Autor } \\
\text { (Ano/ País) }\end{array}$ & Título & Objetivos & $\begin{array}{l}\text { Tipo de } \\
\text { Estudo }\end{array}$ & $\begin{array}{l}\text { Nível de } \\
\text { Evidência }\end{array}$ \\
\hline $\begin{array}{l}\text { Cheruiyot et al. } \\
\qquad(2020)\end{array}$ & $\begin{array}{l}\text { A lesão renal aguda está } \\
\text { associad a pior prognóstico } \\
\text { em pacientes COVID-19: } \\
\text { revisão sistemática e meta- } \\
\text { análise }\end{array}$ & $\begin{array}{c}\text { Esclarecer a associação } \\
\text { clínica entre a gravidade e a } \\
\text { mortalidade do AKI e do } \\
\text { COVID- } 19 .\end{array}$ & $\begin{array}{l}\text { Meta- } \\
\text { análise }\end{array}$ & $\mathrm{I}$ \\
\hline $\begin{array}{l}\text { Costa et al. } \\
\quad(2020)\end{array}$ & $\begin{array}{l}\text { Lesão renal aguda em } \\
\text { pacientes com COVID-19 } \\
\text { de uma UTI no Brasil: } \\
\text { incidência, preditores e } \\
\text { mortalidade hospitalar }\end{array}$ & $\begin{array}{c}\text { O objetivo primário deste } \\
\text { trabalho é identificar a } \\
\text { incidência de LRA e os } \\
\text { possíveis fatores preditores } \\
\text { para sua ocorrência em } \\
\text { pacientes internados com } \\
\text { COVID-19 em UTI de um } \\
\text { hospital privado no Rio de } \\
\text { Janeiro, Brasil; e como objetivo } \\
\text { secundário, avaliar seu impacto } \\
\text { na mortalidade intra-hospitalar. }\end{array}$ & $\begin{array}{l}\text { Estudo de } \\
\text { coorte } \\
\text { retrospecti } \\
\quad \text { vo }\end{array}$ & IV \\
\hline $\begin{array}{l}\text { Fernandes et al. } \\
\qquad(2020)\end{array}$ & $\begin{array}{l}\text { Desafios e recomendações } \\
\text { para o cuidado intensivo de } \\
\text { adultos críticos com } \\
\text { doença de coronavírus } \\
2019\end{array}$ & $\begin{array}{l}\text { Revisar e sintetizar os principais } \\
\text { desafios associados ao cuidado } \\
\text { intensivo do paciente adulto } \\
\text { crítico com COVID-19 e } \\
\text { apresentar evidências de } \\
\text { recomendações. }\end{array}$ & $\begin{array}{c}\text { Revisão } \\
\text { integrativa }\end{array}$ & $\mathrm{V}$ \\
\hline $\begin{array}{l}\text { Fu et al. } \\
(2020)\end{array}$ & $\begin{array}{l}\text { Lesão renal aguda e terapia } \\
\text { de reposição renal no } \\
\text { COVID-19: revisão } \\
\text { sistemática e meta-análise }\end{array}$ & $\begin{array}{l}\text { Fornecer uma estimativa } \\
\text { precisa da incidência de AKI e } \\
\text { KRT em pacientes COVID-19. }\end{array}$ & $\begin{array}{c}\text { Revisão } \\
\text { sistemática }\end{array}$ & I \\
\hline $\begin{array}{l}\text { Gama et al. } \\
\text { (2020) }\end{array}$ & $\begin{array}{l}\text { Pandemia de COVID_19 e } \\
\text { os cuidados de } \\
\text { enfermagem aos pacientes } \\
\text { em tratamento } \\
\text { hemodialítico } \\
\end{array}$ & $\begin{array}{l}\text { Refletir sobre os cuidados de } \\
\text { enfermagem aos pacientes em h } \\
\text { emodiálise no contexto da } \\
\text { pandemia de COVID-19. }\end{array}$ & $\begin{array}{l}\text { Estudo } \\
\text { reflexivo }\end{array}$ & VI \\
\hline $\begin{array}{l}\text { Guedes } \\
(2021)\end{array}$ & $\begin{array}{c}\text { Cuidados de enfermagem } \\
\text { na hemodiálise }\end{array}$ & $\begin{array}{l}\text { Identificar os cuidados de } \\
\text { enfermagem realizados ao } \\
\text { paciente em hemodiálise. }\end{array}$ & $\begin{array}{c}\text { Revisão } \\
\text { integrativa }\end{array}$ & $\mathrm{V}$ \\
\hline $\begin{array}{l}\text { Henry \& Lippi } \\
\text { (2020). }\end{array}$ & $\begin{array}{l}\text { A doença renal crônica está } \\
\text { associada à infecção por } \\
\text { coronavírus grave } 2019 \\
\text { (COVID-19) }\end{array}$ & $\begin{array}{c}\text { A DRC parece estar } \\
\text { associada ao risco aumentado } \\
\text { de infecções graves do } \\
\text { COVID-19. Os pacientes com } \\
\text { DRC devem, portanto, ser } \\
\text { aconselhados a tomar } \\
\text { precauções extras para } \\
\text { minimizar a exposição do risco } \\
\text { ao vírus. }\end{array}$ & $\begin{array}{l}\text { Meta- } \\
\text { análise }\end{array}$ & I \\
\hline $\begin{array}{l}\text { Marinho et al. } \\
\text { (2021) }\end{array}$ & $\begin{array}{l}\text { Assistência de enfermagem } \\
\text { em hemodiálise: (RE) } \\
\text { conhecendo a rotina do } \\
\text { enfermeiro }\end{array}$ & $\begin{array}{c}\text { Conhecer a rotina do } \\
\text { enfermeiro no setor de } \\
\text { hemodiálise de um hospital } \\
\text { público do Nordeste brasileiro. }\end{array}$ & $\begin{array}{l}\text { Artigo } \\
\text { original }\end{array}$ & I \\
\hline
\end{tabular}


Monte et al.

\section{Complicações atípicas e características clínico- epidemiológicas do \\ COVID-19: uma revisão integrativa}

(2020)
Explanar as formas atípicas e características clínico-

epidemiológicas do COVID-19

da COVID-19 através de um

estudo integrativo e abrangente, proporcionando maior entendimento das manifestações clínicas da doença e disponibilizando instrumentos para

embasamento clínico-teórico dos profissionais da saúde.

\begin{tabular}{cc}
\hline & $\begin{array}{c}\text { A atuação do enfermeiro } \\
\text { em unidade de terapia } \\
\text { intensiva na pandemia de } \\
\text { Nunes } \\
\text { COVID-19: relato de } \\
\text { experiência }\end{array}$
\end{tabular}

Relatar a atuação do enfermeiro de uma UTI adulto de pacientes com COVID-19 em hospital público de referência no Sul do país.
Revisão

integrativa

\section{V}

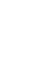

$\begin{array}{ll}\text { Estudo } & \text { VI } \\ \text { descritivo, } & \\ \text { narrativo, } & \\ \text { do tipo } & \\ \text { relato de } & \\ \text { experiênci } & \end{array}$

\begin{tabular}{cc}
\hline & Vista do Atuação da \\
enfermagem no cuidado às \\
Oliveira et al. & pessoas em hemodiálise \\
(2020) & frente à pandemia do vírus \\
& SARS-CoV-2
\end{tabular}
Conhecer as recomendações para guiar a atuação da

a

Revisão

VI

Enfermagem às pessoas com doença renal crônica na prevenção e manejos de casos suspeitos e confirmados de narrativa

COVID-19.

\begin{tabular}{|c|c|c|c|c|}
\hline $\begin{array}{l}\text { Pecly et al. } \\
\text { (2021) }\end{array}$ & $\begin{array}{l}\text { COVID-19 e doença renal } \\
\text { crônica: uma revisão } \\
\text { abrangente }\end{array}$ & $\begin{array}{l}\text { Os desfechos clínicos e } \\
\text { prognóstico de pacientes com } \\
\text { DRC sob tratamento } \\
\text { conservador e diálise, e } \\
\text { receptores de transplante renal } \\
\text { e infecção por COVID-19 }\end{array}$ & $\begin{array}{l}\text { Revisão de } \\
\text { escopo }\end{array}$ & $\mathrm{V}$ \\
\hline $\begin{array}{c}\text { Queiroz \& } \\
\text { Marques (2020) }\end{array}$ & $\begin{array}{c}\text { Gerenciamento de } \\
\text { enfermagem no } \\
\text { enfrentamento da COVID- } \\
19 \text { nos serviços de } \\
\text { hemodiálise }\end{array}$ & $\begin{array}{l}\text { O enfermeiro gestor do } \\
\text { serviço assume as diretrizes } \\
\text { relacionadas a equipe de } \\
\text { enfermagem, ao manejo clínico } \\
\text { dos pacientes e monitoramento } \\
\text { do ambiente físico. }\end{array}$ & $\begin{array}{l}\text { Artigo de } \\
\text { reflexão }\end{array}$ & VI \\
\hline $\begin{array}{l}\text { Saraladevi et al. } \\
\qquad(2020)\end{array}$ & $\begin{array}{l}\text { A epidemia do Novo } \\
\text { Coronavírus } 2019 \text { e rins }\end{array}$ & $\begin{array}{c}\text { Explanar as formas atípicas e } \\
\text { características clínico- } \\
\text { epidemiológicas do COVID-19 } \\
\text { através de um estudo } \\
\text { integrativo e abrangente, } \\
\text { proporcionando maior } \\
\text { entendimento das } \\
\text { manifestações clínicas da } \\
\text { doença e disponibilizando } \\
\text { instrumentos para } \\
\text { embasamento clínico-teórico } \\
\text { dos profissionais da saúde. }\end{array}$ & $\begin{array}{c}\text { Revisão } \\
\text { integrativa }\end{array}$ & $\mathrm{V}$ \\
\hline
\end{tabular}
COVID-19: uma nova
ameaça para a prática da nefrologia e como

Ulu et al.

Esse amplo espectro de
influência do COVID-19 sobre
pacientes renais e funções
renais obviamente necessitam

Revisão

narrativa

renais obviamente necessitam com

natureza 


\begin{tabular}{|c|c|c|c|}
\hline$(2020)$ & pequena devastação? & $\begin{array}{l}\text { de uma política de gestão } \\
\text { atualizada para o cuidado } \\
\text { nefrológico. }\end{array}$ & qualitativa \\
\hline $\begin{array}{c}\text { Wang et al. } \\
(2020)\end{array}$ & $\begin{array}{l}\text { Curso clínico e resultado de } \\
107 \text { pacientes infectados } \\
\text { com o novo corona vírus, } \\
\text { SARS- CoV-2, teve alta de } \\
\text { dois hospitais em Wuhan, } \\
\text { China }\end{array}$ & $\begin{array}{l}\text { Descrever os cursos clínicos } \\
\text { e prognósticos em pacientes } \\
\text { COVID-19. }\end{array}$ & $\begin{array}{l}\text { Estudo } \\
\text { retrospecti } \\
\text { vo }\end{array}$ \\
\hline
\end{tabular}

Fonte: Elaboração dos autores, 2021.

\section{DISCUSSÃO}

Foi possível constatar que o enfermeiro contribui desde a participação no estabelecimento do diagnóstico de LRA a implementação de suas medidas terapêuticas. Avaliando criteriosamente o paciente, prescrevendo cuidados de enfermagem afim de proporcionar uma assistência individualizada, participando ativamente do processo de tomada de decisões junto à equipe multidisciplinar. Atuando ainda na organização, supervisão e direção dos serviços de enfermagem, atentando-se para vigilância e identificação precoce de eventos adversos contribuindo na gestão assistencial.

Os pacientes críticos com COVID-19 manifestam uma sintomatologia variada, em sua maioria desencadeando danos pulmonares, podendo originar complicações como lesão renal englobando também falência múltipla de órgãos. Salienta-se que a LRA nesses casos possui relação com a infecção ocasionada pelo coronavírus associados a fatores como idade avançada, diabetes e hipertensão que elevam o risco de progredir para uma LRA (Fernandes et al., 2020; Fu et al., 2020). Segundo Nunes (2020), essas lesões têm acometido pacientes com COVID-19 de maneira frequente, aproximadamente 40\% destes na admissão hospitalar desenvolvem LRA. Dentre este grupo, 20\% necessitam de terapia de substituição renal (TSR) ao decorrer de sua evolução clínica, constatando uma taxa de mortalidade em torno de 30\%.

Nesse contexto, as lesões renais evidenciam um início com alterações leves podendo debilitar para uma deterioração. O desenvolvimento de lesão renal em indivíduos acometidos por COVID-19 exprime como determinante para um pior curso da doença, abrangendo maior risco de gravidade e mortalidade, especialmente, aos pacientes com Lesão Renal Crônica (LRC) em decorrência da supressão imunológica, favorecendo um prognóstico desfavorável. Em consequência disso, ambos os quadros possuem vulnerabilidade a infecções oportunistas, especialmente os pacientes que precisam de diálise (Cheruiyot et al., 2020; Monte et al., 2020; Saraladevi et al., 2020).

Dentre os agravos decorrentes da infecção pelo novo coronavírus, é considerado que a ocorrência de LRA em pacientes acometidos, sobrevém pela correlação já conhecida entre a Síndrome do Desconforto Respiratório Agudo (SRDA) com lesão renal proveniente dos danos pulmonares pré-existentes e da frequente utilização de suporte ventilatório. Nessa circunstância, estudos realizados em centros brasileiros e europeus 
retratam que a incidência de LRA eleva-se em 20\% a 50\% em pacientes que fazem uso de ventilação mecânica por COVID-19 grave (SBN \& AMIB, 2020).

É relatado que a COVID-19 atinge múltiplos sistemas corporais, portanto, um prognóstico positivo em pacientes que apresentam LRA e COVID-19 simultaneamente, requer a contenção de diversos fatores desencadeantes dessa patologia. Uma vez que, a falência renal pode acarretar disfunções orgânicas importantes, assim como alterar o equilíbrio metabólico. Dessa forma, esse quadro pode gerar alterações como letargia, desorientação ou torpor, os quais tem potencial de ocorrerem em função de hipoxemia ou acidose no paciente (Monte et al., 2020; Saraladevi et al., 2020).

Os mecanismos do envolvimento renal ainda não estão esclarecidos com exatidão, assim como o seu tratamento, porém, mostra-se complexa e multifatorial. Toda via, é observado que independentemente da fisiopatologia implícita, é notório que a ocorrência de LRA possui relação com o COVID-19 e está associada a uma pior progressão clínica e/ou mortalidade do indivíduo. Diante disso, é evidente que a assistência prestada a esse público concerne cuidados intensivos em uma UTI, ambiente este complexo que demanda preparação e disponibilidade de uma equipe multiprofissional participativa, e frente a isso maior envolvimento da equipe de enfermagem (Cheruiyot et al., 2020; Henry \& Lippi, 2020; Saraladevi et al., 2020).

As UTI's visam o atendimento a pacientes com gravidade acentuada e que requerem uma assistência integral, juntamente ao uso de equipamentos e dispositivos especializados que a mesma dispõe. A ampla frequência de pacientes com LRA nas UTI's, diligenciam do enfermeiro uma assistência com qualidade e segurança. Implicando um quadro de dimensionamento adequado dos profissionais treinados e capacitados para atender os cuidados necessários aos pacientes com LRA, bem como o gerenciamento de recursos e insumos (Nunes, 2020; Oliveira et al., 2020).

Guedes et al. (2021) reitera que mesmo com a realidade de avanços na tecnologia, as lesões renais agudas ainda são uma das síndromes preponderantes que mais afetam pacientes hospitalizados. Mediante a situação pandêmica declarada pela OMS no ano de 2020 em decorrência de contaminações ocasionadas pelo SarsCov-2, o quantitativo de pacientes em UTI's obteve um crescimento significativo nesse período. Nessa condição, é possível caracterizar a ocorrência de LRA em pacientes contaminados pelo COVID-19, como um agravo em decorrência da infecção.

Frente a esse cenário de complexidade, é convincente a notoriedade da enfermagem nos sistemas de saúde no brasileiro e mundial, pois, estes consolidam o domínio de técnicas e conhecimentos científicos baseado em evidências. Atuando na coordenação do cuidado, além da direção e organização de suas atividades técnicas e auxiliares, firmando um comprometimento com o paciente o qual recebe a assistência, que por sua vez deve ser qualificada, atendendo as necessidades a ele intrínsecas (Gama et al., 2020).

É importante considerar também que a enfermagem atua com papel central no desenvolvimento dos cuidados aos pacientes que estão em tratamento dialítico, por meio de intervenções biopsicossociais baseadas nos pilares assistenciais que atendam a esse público. Apresentando como finalidade a promoção do bem-estar, 
através da busca conjunta de melhorar a qualidade de vida destes que manifestam essa condição, não somente conferindo a equipe competências técnicas (Guedes et al., 2021).

No desempenho de suas funções, os enfermeiros juntamente aos demais profissionais da saúde comprometidos com a assistência nefrológica devem conceder os cuidados necessários da melhor forma aos pacientes pelos quais são responsáveis. Exercendo práticas que atenuem ao máximo os riscos de auto contaminação e consequentemente estendendo esses cuidados aos seus pacientes. Atentando-se para padronização do uso adequado dos equipamentos de proteção individual (EPI), implementando protocolos voltados para a circulação das equipes no setor em que se encontram pacientes acometidos por COVID-19 por exemplo, com a finalidade de limitar o deslocamento desnecessário nos ambientes destinados ao isolamento desses (Nunes, 2020SBN \& AMIB, 2020).

Não obstante, observa-se por meio de um estudo realizado em uma unidade de hemodiálise em um hospital na cidade de Campina Grande, no estado da Paraíba, que as numerosas atribuições na rotina do enfermeiro nesse setor associados a carga de trabalho e carência de apoio de uma equipe multiprofissional mais atuantes são elencadas como barreiras no gerenciamento do cuidado. Resultando em uma alta carga de estresse e sentimentos de incompetência para esses profissionais, provenientes do cuidado direto aos pacientes. Fatores estes decorrentes que demandam em sua maioria competências especializadas das demais áreas inseridas na assistência (Marinho et al., 2021).

Com o objetivo de atenuar essas condições vivenciadas pelos enfermeiros ressalta-se a importância desses profissionais conciliarem as atividades práticas e administrativas baseadas nos princípios organizacionais e de planejamento com foco principal na promoção da qualidade e segurança prestada neste cenário. Tonando-se imprescindível a participação conjunta de outros profissionais na busca pela garantia da integralidade do cuidado no atendimento voltado às clínicas críticas e suas respectivas comorbidades, bem como aos possíveis riscos à saúde inerentes ao período de internação (Nunes, 2020; Marinho et al., 2021).

Corroborando essas ações, vale ressaltar que é um direito assegurado e um dever empregado aos enfermeiros o aperfeiçoamento de seus conhecimentos e habilidades técnico-científicas. Com isso, ao aprimorar seus fundamentos, têm-se como propósito alicerçar com segurança e qualidade a assistência ofertada àqueles que demandam da atenção desse profissional. No entanto, ao mesmo tempo que se faz necessário adquirir e atualizar os saberes, o desenvolvimento gradativo da qualificação do enfermeiro intensivista é um dos maiores impasses devido a privação de tempo, estresse, cansaço e dificuldade em conciliar o processo educativo com os compromissos de trabalho (Cofen, 2017; Nunes, 2020).

Os obstáculos supracitados são intensificados pela circunstância atual vivenciada em decorrência da pandemia. Dada essa conjuntura, salienta-se a necessidade de implementar ações de cunho educativo em saúde, como os programas de educação permanente, especialmente quando estabelecidas pelas instituições em que o enfermeiro está inserido. Propiciando desta forma ao profissional meios facilitadores de aprimoramento do 
processo de qualificação de suas atividades, beneficiando ambos e consequentemente influenciando na assistência ofertada aos pacientes (Gama et al., 2020; Marinho et al., 2021).

As limitações deste estudo estão relacionadas a carência de produções científicas relacionadas à temática, visto que a abordagem pertinente ao COVID-19 nesse contexto ainda é recente. Dessa forma, a produção de estudos pertinentes as pontuações citadas tornam-se relevantes para esclarecimentos subsequentes da associação que a LRA possui com COVID-19 e a atuação do enfermeiro nesse contexto. A fim de que a enfermagem possa contribuir com maior diligência e habilidades técnico-científicas à assistência necessária.

\section{CONSIDERAÇÕES FINAIS}

A construção do presente estudo permitiu importantes contribuições no que diz respeito aos cuidados prestados pelo enfermeiro ao paciente com LRA pós COVID-19 em uma UTI, corroborando juntamente na identificação dos obstáculos enfrentados na conjuntura vivenciada. Fomentando a necessidade de refletir acerca das condições de trabalho, sejam elas materiais ou não, a valorização e a importância das práticas educacionais voltadas à capacitação continuada, o que interfere positiva e diretamente no cuidado direcionado ao paciente.

Foi possível ainda observar o crescimento acelerado em relação ao número de casos de indivíduos que desenvolveram LRA desencadeada pela infecção por COVID-19, associada também a demais fatores préexistentes. Em virtude ao quadro de vulnerabilidade que implica essa patologia, ficou evidente a relevância do papel desempenhado pelo profissional enfermeiro centrado no cuidado físico abrangendo similarmente fatores biopsicossociais, na busca pela promoção da saúde e bem-estar do paciente no contexto inserido.

As lacunas identificadas na assistência a este público reforçam a necessidade de reorganização no processo de trabalho da equipe de enfermagem alinhados as atribuições de uma equipe multiprofissional atuante. Com intuito de reduzir a sobrecarga de trabalho enfrentada pelos enfermeiros, possibilitando estes a qualificação e lapidação do seu processo de trabalho.

\section{CONFLITOS DE INTERESSES}

Os autores declaram que não há conflitos de interesse. Todos os autores estão cientes da submissão do artigo.

\section{REFERÊNCIAS}

Brasil. Ministério da Saúde. Secretaria de Atenção Especializada à Saúde (SAES) Departamento de Atenção Hospitalar, Domiciliar e de Urgência (DAHU). (2020). Protocolo de tratamento do novo coronavírus (2019CoV). Brasília: Ministério da Saúde.

Cheruiyot, I., Kipkorir, V., Ngure, B., Misiani, M., Munguti, J., Henry, B. \& Lippi, G. (2020). A lesão renal aguda está associada pior prognóstico em pacientes COVID-19: revisão sistemática e meta-análise. Acta Biomed. 91 (3), $1-19$. 
Cordeiro, L. \& Soares, C. B. (2019). Revisão de escopo: potencialidades para a síntese de metodologias utilizadas em pesquisa primária qualitativa. Boletim do Instituto de Saúde. 2 (20), 37-43.

Costa, R. L., Sória, T. C., Salles, E. F., Gerecht, A. V., Corvisier, A. F., Menezes, M. A. M., Ávila, C. S., Silva, E. C., F., Silva, E. C., Pereira, S. R. \& Simvoulidis, L. F. (2021). Lesão renal aguda em pacientes com COVID-19 de uma UTI no Brasil: incidência, preditores e mortalidade hospitalar. Bra\%. J. Nephrol. (J. Bras. Nefrol.). 43 (3), 349358.

Departamento de Informática do SUS - DATASUS. (2019). Procedimentos Hospitalares do SUS.

Fernandes, C. A., Cunha, A. M., Hemkemaier, E. C., Carmo, K. S., Moraes, T. V., Santos, T. P., Silva, T. P., Melo, L. E. \& Mateus, N. C. (2020). Desafios e recomendações para o cuidado intensivo de adultos críticos com doença de coronavírus 2019. Health Residencies Journal (HRJ). 1 (1), 1-26.

Fu, E. L., Janse, R. J., Jong, Y., Endt, V. H., Milders, J., Willik, E. M., Rooij, E. N., Dekkers, O. M., Rotmas, J. L. \& Diepen, M. V. (2020). Lesão renal aguda e terapia de reposição renal no COVID-19: revisão sistemática e meta-análise. Clinical Kidney Journal. 12 (4), 550-563.

Gama, B. M., Cruz, C. M., França, L. M., Ferreira, M. R., Gomes, S. S. \& Godinho, M. R. (2020). Pandemia de COVID_19 e os cuidados de enfermagem aos pacientes em tratamento hemodialítico. Escola Anna Nery. 24 (spe) $1-7$.

Guedes, J. B., Lacerda, M. R., Nascimento, J. D., Tonin, L. \& Caceres, T. G. (2021). Cuidados de enfermagem na hemodiálise. Rev. Pesq. Cuidado é Fundamental. 13, (9402), 653-660.

Henry B. M., \& Lippi G. (2020). Chronic kidney disease is associated with severe coronavirus disease 2019 (COVID-19) infection. Int Urol Nephrol. 52 (6), 1193-4.

Marinho, I. V., Santos, D. G., Bittelbrunn, C., Carvalho, A. L., Vasconcelos, N. C. \& Silva, M. L. (2021). Assistência de enfermagem em hemodiálise: (RE) conhecendo a rotina do enfermeiro. Enfermagem em Foco. 12 (32), 354-359.

Monte, L. M., Mendes, L. A., Camargo, R. L., Gomes, R. S., Silveira, P. H., Seyfarth, M. S., Cunha, D. M., Oliveira, L. P., Silveira, R. \& Silveira, G. R. (2020). Complicações atípicas e características clínicoepidemiológicas do COVID-19: uma revisão integrativa. Revista Eletrônica Acervo Saúde. Esp.(46), 1-12.

Nunes, M. R. A atuação do enfermeiro em unidade de terapia intensiva na pandemia de COVID-19: relato de experiência. (2020). Revista Eletrônica Acervo Saúde / Electronic Journal Collection Health. 12 (11), 1-6.

Oliveira, N. P., Schwartz, D. S., Spagnolo, D., Cunha, T. N., Neves, J. L. \& Lise, F. (2020). Vista do Atuação da enfermagem no cuidado às pessoas em hemodiálise frente à pandemia do vírus SARS-CoV-2. Enfermagem Brasil. 19 (4), 26-33.

Pecly, I. M., Azevedo, R. B., Muxfeldt, E. S., Botelho, B. G., Albuquerque, G., Diniz, P. H., Silva, R. \& Rodrigues, C. I. S. (2021). COVID-19 e doença renal crônica: uma revisão abrangente. Brą. J. Nephrol. (J. Bras. Nefrol.). 43 (3), 383-39.

Peters, M. D. J. et al. (2020). Chapter 11: Scoping Reviews (2020 version). In: Aromataris E, Munn Z (Editors). JBI Manual for Evidence Synthesis. Joanna Briggs Institute.

Queiroz, Z. J. \& Marques, P. F. (2020). Gerenciamento de enfermagem no enfrentamento da COVID-19 nos serviços de hemodiálise. Enfermagem em Foco. 11 (1), 196-198. 
Cofen. Resolução no 564/2017 (BR). (2017). Aprova o novo Código de Ética dos Profissionais de Enfermagem. Diário Oficial da União. Brasília (DF).

Saraladevi, N., Chih-Wei, Y., Shang- Jyn, H., Bi-Cheng, L., Jiang-Hua, C. \& Vivekanand, J. (2020). A epidemia do Novo Coronavirus 2019 e rins - Kidney International (kidney-international.org) National Center for Biotechnology Information. 97 (5), 824-828.

Sociedade Brasileira de Nefrologia - SBN \& Associação de Medicina Intensiva Brasileira. (2020). Nota técnica e orientações sobre a injúria renal aguda (IRA) em pacientes com COVID-19.

Ulu, S., Gungor, O., Gok Oguz, E., Hasbal, N. B., Turgut, D., \& Arici, M. (2020). COVID-19: a novel menace for the practice of nephrology and how to manage it with minor devastation?. Renal failure. 42 (1), 710-725.

Wang, D., Yin, Y., Hu, C., Liu, X.., Zhang, X. \& Zhou S. (2020). Clinical course and outcome of 107 patients infected with the novel coronavirus, SARS-CoV-2, discharged from two hospitals in Wuhan, China. Crit Care. 24 (188), 1-9.

\section{Contribuições dos autores}

Todos os autores contribuíram com a coleta dos dados, escrita e revisão do trabalho. 